\title{
Essentials for Selecting Antimicrobial Therapy for Intra-Abdominal Infections
}

\author{
Stijn Blot, Jan J. De Waele and Dirk Vogelaers
}

Faculty of Medicine \& Health Sciences, Ghent University, Ghent, Belgium

\section{Contents}

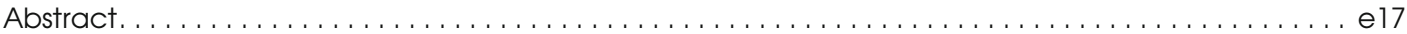

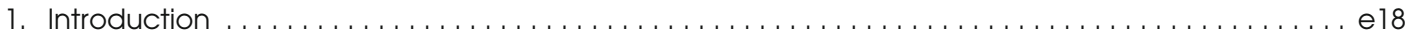

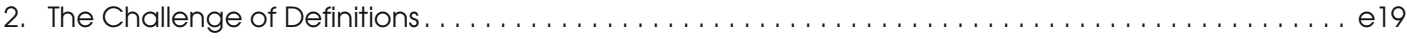

2. 1 Primary, Secondary and Tertiary Peritonitis/Complicated vs Non-Complicated

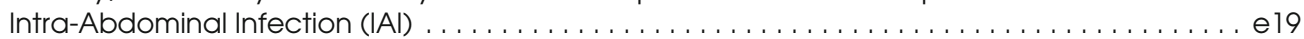

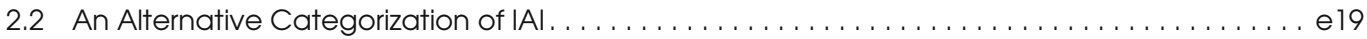

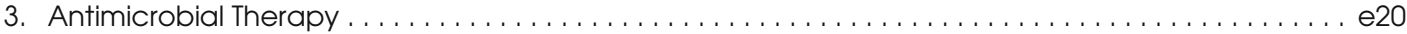

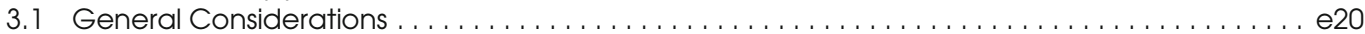

3.2 Factors to Take into Account when Selecting Empirical Antimicrobial Therapy for IAI . . . . . . e21

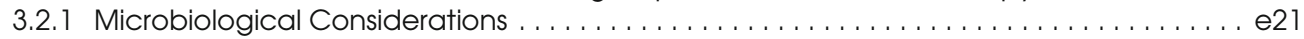

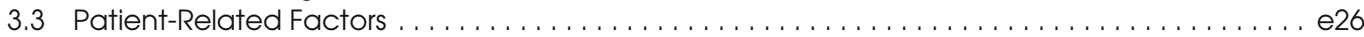



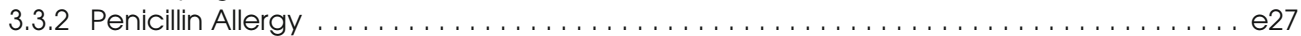

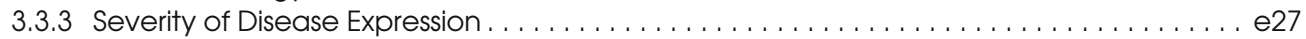

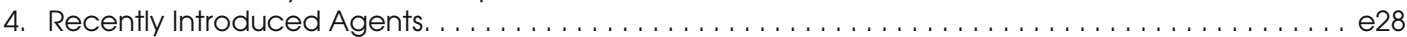





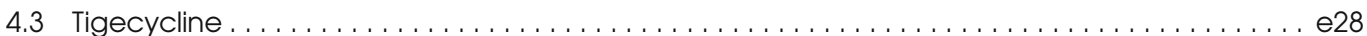

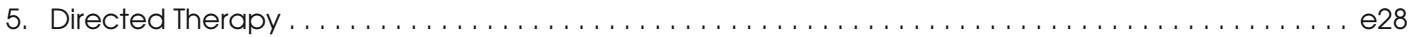

6. Duration of Antimicrobial Therapy for IAI . . . . . . . . . . . . . . . . . . . . . . . . . e29

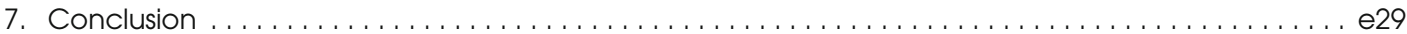

\section{Abstract}

Intra-abdominal infection (IAI) is a complex disease entity in which different aspects must be balanced in order to select the proper antimicrobial regimen and determine duration of therapy. A current classification indicates different faces of peritonitis. Primary peritonitis implies an intact gastrointestinal tract without overt barrier disruption. Secondary peritonitis refers to localized or diffuse peritoneal inflammation and abscess formation due to disruption of the anatomical barrier. Tertiary peritonitis includes cases that cannot be solved by a single or even sequential surgical intervention, often in combination with sequential courses of antimicrobial therapy. The most frequently used classification distinguishes 'uncomplicated' and 'complicated' IAI. In uncomplicated IAI, the infectious process is contained within a single organ, without anatomical disruption. In complicated IAI, disease is extended, with either localized or generalized peritonitis. However, there exists more than a single dimension of complexity in IAI, including severity of 
disease expression through systemic inflammation. As the currently used classifications of IAI often incite confusion by mixing elements of anatomical barrier disruption, severity of disease expression and (the likelihood of) resistance involvement, we propose an alternative for the current widely accepted classification. We suggest abandoning the terms 'uncomplicated' and 'complicated' IAI, as they merely confuse the issue. Furthermore, the term 'tertiary peritonitis' should likewise be discarded, as this simply refers to treatment failure of secondary peritonitis resulting in a state of persistent infection and/or inflammation. Hence, anatomical disruption and disease severity should be separated into different phenotypes for the same disease in combination with either presence or absence of risk factors for involvement of pathogens that are not routinely covered in first-line antimicrobial regimens (Pseudomonas aeruginosa, enterococci, Candida species and resistant pathogens). Generally, these risk factors can be brought back to recent exposure to antimicrobial agents and substantial length of stay in healthcare settings (5-7 days). As such, we developed a grid based on the different components of the classification: (i) anatomical disruption; (ii) severity of disease expression; and (iii) either community-acquired/early-onset healthcare-associated origin or healthcare-associated origin and/or recent antimicrobial exposure. The grid allows physicians to define the index case of IAI in a more unequivocal way and to select the most convenient empirical antimicrobial regimens. The grid advises on the necessity of covering nosocomial Gram-negative bacteria (including P. aeruginosa), enterococci and yeasts. The basis of antimicrobial therapy for IAI is that both Gram-negative and anaerobic bacteria should always be covered.

In recent years, some newer agents such as doripenem, moxifloxacin and tigecycline have been added to the antimicrobial armamentarium for IAI. For patients in whom the source can be adequately controlled, antimicrobial therapy should be restricted to a short course (e.g. 3-7 days in peritonitis).

\section{Introduction}

Intra-abdominal infections (IAI) represent a particular clinical challenge, as they differ from other types of infections in a number of aspects. ${ }^{[1]}$ The clinical spectrum of IAI is very broad, ranging from uncomplicated acute appendicitis to generalized peritonitis caused by a perforated ischaemic bowel. Surgery - often referred to as surgical source control - is essential and generally considered to be a decisive factor for the outcome in patients with IAI. ${ }^{[2-4]}$ Source control is defined as the elimination of the source of infection and the control over ongoing contamination. This concept is based on the following principles: drainage of abscesses or contaminated fluid, debridement of necrotic tissue, and restoration of anatomy and function. ${ }^{[5]}$ In addition, during surgery for gastrointestinal pathology, excision of diseased viscus, if feasible, and gastrointestinal diversion, if applicable, may limit the risk of anastomotic leakage and subsequent peritonitis. Source control may be achieved through laparotomy, laparoscopy and percutaneous drainage (ultrasound or CT guided). In the absence of perforation, operative intervention is generally not indicated.

The microbial aetiology and microbiological diagnosis pose another challenge. As the gastrointestinal tract contains a huge number of different micro-organisms, IAI is usually polymicrobial. Yet, not all bacteria can be isolated by means of routine cultures. ${ }^{[6]}$ On the other hand, not all bacteria isolated represent true pathogens, as most enteric bacteria are of relatively low virulence. 
The profile of the patient determines the approach for IAI. Community-acquired IAI may be more rapidly diagnosed than that occurring in critically ill patients in whom signs and symptoms may be masked by other inflammatory processes. Critically ill patients also have more pronounced risk profiles, as IAI is more often associated with acute kidney injury and septic shock. ${ }^{[7]}$ Furthermore, a healthcare origin of infection is important as there exists a higher likelihood of involvement of resistant pathogens with the potential for inappropriate empiric antimicrobial therapy and detrimental outcomes. In the present review, emphasis is given to empirical choices of antimicrobial therapy in patients with IAI. Special attention is given to the different elements that should be taken into account in order to select the proper regimen. Issues such as new agents and duration of therapy are mentioned briefly.

\section{The Challenge of Definitions}

\subsection{Primary, Secondary and Tertiary}

Peritonitis/Complicated vs Non-Complicated Intra-Abdominal Infection (IAI)

Primary peritonitis includes distinct disease entities such as peritonitis in decompensated liver cirrhosis and peritonitis in nephrotic syndrome with ascites (e.g. with Streptococcus pneumoniae). These imply an intact gastrointestinal tract without overt barrier disruption. Secondary peritonitis refers to localized or generalized peritoneal inflammation and abscess formation due to disruption of the anatomical barrier (e.g. perforated appendicitis or diverticulitis). In the literature, so-called 'tertiary peritonitis' has been defined, referring to cases that cannot be solved by a single or even sequential surgical intervention, in combination with often sequential courses of antimicrobial therapy, either with or without antifungal coverage. ${ }^{[8]}$ A suggested approach for these difficult-to-solve cases consisted of an open-abdomen treatment associated with chronic serositis and colonisation with often resistant pathogens. This definition refers to treatment failure in secondary peritonitis.

The most frequently used classification for IAI in randomized controlled trials of antimicrobial regimens distinguishes between 'uncomplicated' and 'complicated' IAI. ${ }^{[9]}$ In uncomplicated IAI, the infectious process is contained within a single organ, without anatomical disruption. In most patients, these infections can be managed with surgical resection alone and, with the exception of peri-operative prophylaxis, no additional antimicrobial therapy is necessary. Acute appendicitis and cholecystitis are common examples of uncomplicated IAI.

In 'complicated' IAI, disease is extended with either localized or generalized peritonitis. These cases require a more prolonged course of antimicrobials following the surgical procedure.

In clinical trials, essentially only IAI with uneventful clinical courses are selected. Infections are categorized as complicated on the basis of the sole criterion of anatomical barrier disruption, and selected on the basis of often rigid inclusion and exclusion criteria for cases with mild to moderate disease severity. However, IAI comprises more than a single dimension of complexity. In the mind of clinicians, 'complicated' may refer to severity of disease expression, anatomical extent of the infectious process and the involvement of resistant pathogens. Hence, the literature has been contaminated with this bias.

\subsection{An Alternative Categorization of IAI}

As the current classifications of IAI often incite confusion by mixing elements of anatomical barrier disruption, severity of disease expression and (the likelihood of) resistance involvement, we propose an alternative. We suggest abandoning the terms 'uncomplicated' and 'complicated' IAI, as they merely confuse the issue. Furthermore, the term 'tertiary peritonitis' should likewise be discarded, as this simply refers to treatment failure of secondary peritonitis resulting in a state of persistent infection and/or inflammation. Hence, anatomical disruption and disease severity should be separated into different phenotypes for the same disease (e. g. diverticulitis); this should then be combined with either presence or absence of risk factors for involvement of pathogens that are not routinely covered in first-line antimicrobial regimens (Pseudomonas aeruginosa, enterococci, 
Table I. Classification of intra-abdominal infections

\begin{tabular}{|c|c|c|c|}
\hline & \multicolumn{3}{|c|}{ Disease expression } \\
\hline & $\begin{array}{l}\text { Mild } \\
\text { (sepsis) }\end{array}$ & $\begin{array}{l}\text { Moderate } \\
\text { (severe sepsis) }\end{array}$ & $\begin{array}{l}\text { Severe } \\
\text { (septic shock) }\end{array}$ \\
\hline \multicolumn{4}{|c|}{$\begin{array}{l}\text { Community-acquired or early-onset healthcare-associated IAI } \\
<7 \text { days after hospital admission) }\end{array}$} \\
\hline Without perforation & 1 & 1 & 2 \\
\hline Localized peritonitis & 1 & 1 & 2 \\
\hline Diffuse peritonitis & 1 & 2 & 2 \\
\hline \multicolumn{4}{|c|}{$\begin{array}{l}\text { Late-onset healthcare-associated IAI ( } \geq 7 \text { days after hospital } \\
\text { admission) and/or recent antimicrobial exposure }\end{array}$} \\
\hline Without perforation & 2 & 2 & 2 \\
\hline Localized peritonitis & 2 & 2 & 3 \\
\hline Diffuse peritonitis & 2 & 3 & 3 \\
\hline
\end{tabular}

Candida species and resistant pathogens). Generally, these risk factors can be reduced to recent exposure to antimicrobial agents and substantial length of stay in healthcare settings (5-7 days). As such, we developed a grid based on the different components in the classification: (i) anatomical disruption; (ii) severity of disease expression; and (iii) either community-acquired/early-onset healthcare-associated origin or late-onset healthcareassociated origin and/or recent antimicrobial exposure (table I).

The grid allows physicians to define the index case of IAI in a more unequivocal way and subsequently to select the most convenient empirical antimicrobial regimen (table II). As IAI basically always requires coverage against Gram-positive, Gram-negative and anaerobic bacteria, the grid more precisely advises on the necessity of covering nosocomial Gram-negative bacteria (including $P$. aeruginosa), enterococci, yeasts and resistant pathogens. The necessity of additionally covering these pathogens is based on the assessment of overall risk of treatment failure (according to the three components of the IAI classification). For example, the grid advises a baseline regimen for community-acquired IAI presenting with localized peritonitis and severe sepsis. However, should the patient present with either diffuse peritonitis and/or septic shock, the grid advises to additionally cover nosocomial Gram-negative bacteria and enterococci. This is because it is perceived as a grim clinical situation (more diffi- cult surgical cleaning and/or presence of organ failure) in which the negative consequences of inappropriate antimicrobial therapy are close to inevitable. However, from a purely microbiological viewpoint, there is little to support this approach. The antimicrobial options provided by the grid are mainly based on clarity and simplicity, albeit however, that local epidemiology should always be considered as well (see section 3.2.1). The grid does not include the additional dimension of treatment failure. As such, it reflects the initial conditions of IAI presentation, similar to a severity of disease scoring system used as a prognostic indicator such as the APACHE II (Acute Physiology and Chronic Health Evaluation II) score reflecting the first 24 hours of disease presentation. ${ }^{[10]}$ However, throughout this paper, we use the definitions used in individual publications.

\section{Antimicrobial Therapy}

\subsection{General Considerations}

The main objectives of antimicrobial therapy in the treatment of IAIs are to prevent local and haematogenous spread, and to reduce late complications. As for other infections, early administration of antibacterials is important, and it is logical that these are administered upon the diagnosis of complicated IAI. When preoperative imaging is unequivocal, treatment should not be delayed for intraoperative confirmation. Perioperative prophylaxis should not be used as an alternative or temporizing strategy in these patients: for patients with suspected IAI but equivocal findings who undergo explorative laparotomy, we advise the administration of antibacterials even without confirmation of IAI; when clinical syndrome and preoperative imaging are highly suggestive, treatment should not be delayed for intra-operative confirmation. This is a setting of early empirical treatment requiring high sensitivity for the diagnosis of complicated IAI at a certain cost for specificity, and not a setting of peri-operative prophylaxis. In critically ill patients, the threshold for starting antibacterials may be even lower, as the delayed treatment of infections is associated with considerable morbidity and 


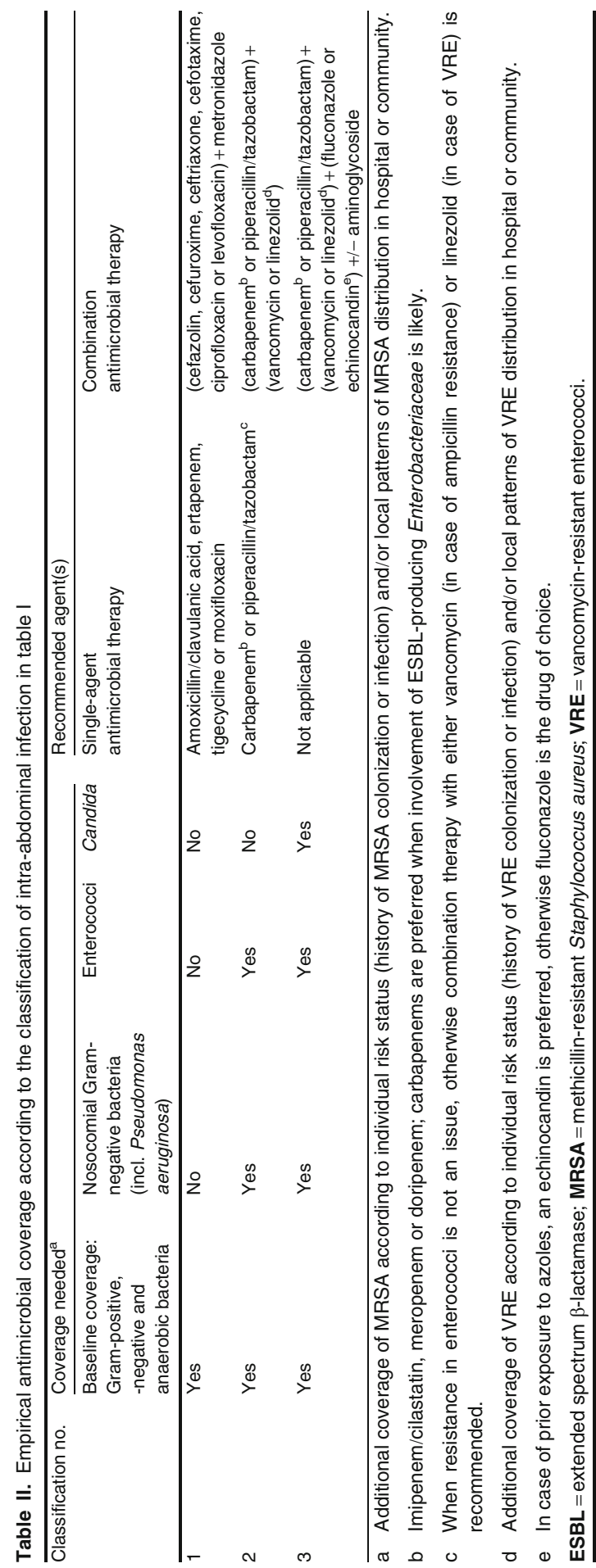

mortality. Upon documentation of intra-abdominal pathology without infection, antibacterials should be discontinued.

Intravenous administration is the only route for early antimicrobial treatment in truly complicated IAI. Oral administration is to be avoided at that stage, as absorption will usually be unreliable, and the effect may be delayed. Postoperatively, oral administration may be considered with improvement of clinical condition and recovery of gastrointestinal function.

\subsection{Factors to Take into Account when Selecting Empirical Antimicrobial Therapy for IAI}

IAI are typically polymicrobial, and require empirical coverage for Gram-positive and Gramnegative, aerobic and anaerobic bacteria, with the latter most relevant for infections originating from the lower gastrointestinal tract (distal small bowel to rectum). Whether, in addition, coverage must be given for enterococci, $P$. aeruginosa, Candida species and resistant pathogens, such as extended-spectrum $\beta$-lactamase (ESBL)-producing Enterobacteriaceae, depends on microbiological issues, epidemiological patterns and patientrelated factors.

\subsubsection{Microbiological Considerations}

The microbiology of IAI reflects a summary of transient or persistent normal gastrointestinal flora with potentially pathogenic micro-organisms. As such, the aetiology of IAI includes Grampositive, Gram-negative and anaerobe bacteria as well as fungal species. However, about $20-25 \%$ of cultures in secondary peritonitis prove negative. ${ }^{[11]}$ In another quarter of the patients, the infection is monomicrobial and, in half of the patients peritonitis is polymicrobial. ${ }^{[12]}$ However, the precise mix of pathogens involved is highly variable depending on several factors that lead to the risk profile of the patient. Table III gives an overview of the most common causative pathogens in IAI. Interpretation of the extent of microorganisms involved is hampered by the limited microbiological workup of anaerobe cultures in general practice. In principle, IAI are polymicrobial with an aerobic component (inducing 
Table III. Frequently isolated pathogens in complicated intra-abdominal infections

\begin{tabular}{|c|c|}
\hline Micro-organism & Clinically relevant resistance problem \\
\hline \multicolumn{2}{|l|}{ Gram-negative bacteria } \\
\hline $\begin{array}{l}\text { Enterobacteriaceae (Escherichia coli, Enterobacter } \\
\text { spp., Klebsiella spp., Proteus spp., etc. ) }\end{array}$ & $\begin{array}{l}\text { ESBL-producing strains likely in healthcare-associated infection. In E. coli } \\
\text { fluoroquinolone-resistance may be up to } 20 \% \text { in some geographic areas }\end{array}$ \\
\hline $\begin{array}{l}\text { Non-fermenting Gram-negative bacteria ( } P \text { seudomonas } \\
\text { aeruginosa, Acinetobacter baumannii, etc.) }\end{array}$ & Multidrug resistance most likely in healthcare-associated infection \\
\hline \multicolumn{2}{|l|}{ Gram-positive bacteria } \\
\hline Streptococci & No clinically relevant resistance problem. \\
\hline Enterococci & $\begin{array}{l}\text { Problems of resistance likely in healthcare-associated infections, especially } \\
\text { when caused by Enterococcus faecium. }{ }^{[13]} \text { Ampicillin-resistance and associated } \\
\text { production of } \beta \text {-lactamases is a concern in some geographic areas, as is } \\
\text { glycopeptide resistance }\end{array}$ \\
\hline Coagulase-negative Staphylococci & $\begin{array}{l}\text { Methicillin resistance most likely in healthcare-associated infection. Clinical } \\
\text { pathogenecity uncertain }\end{array}$ \\
\hline $\begin{array}{l}\text { Anaerobic bacteria (Bacteroides fragilis, } \\
\text { Clostridium spp., etc) }\end{array}$ & $\begin{array}{l}\text { Important resistance against clindamycin and cefoxitin in certain geographic } \\
\text { areas. Resistance against metronidazole is rare }{ }^{[14]}\end{array}$ \\
\hline Candida spp. & $\begin{array}{l}\text { Selection towards non-albicans Candida spp. with reduced susceptibility to } \\
\text { fluconazole in patients with prior exposure to this agent in hospitalized patients }\end{array}$ \\
\hline
\end{tabular}

local/systemic inflammation) and an anaerobic component (responsible for abscess formation).

The value of microbiological identification is a matter of debate, as cultures often reveal mixed flora in which it is difficult to distinguish contaminants from true pathogens. In cases of community-acquired IAI with secondary peritonitis, cultures rarely influence the management of the patient as the encountered flora is generally susceptible to the standard regimens. ${ }^{[15]}$ In cases of healthcare-associated IAI, peri-operative culturing is routinely indicated, as responsible pathogens are less predictable and more likely resistant to first-line or standard empirical regimens (amoxicillin/clavulanic acid or combination of cefuroxim plus a 5-nitroimidazole). In addition, peri-operative cultures may reveal other pathogens such as enterococci, $P$. aeruginosa or yeast, which are also not routinely covered by first-line antimicrobial regimens. As such, culture results allow for either correction of an initially inappropriate choice or de-escalation. However, irrespective of the isolated pathogens, coverage against Grampositive, Gram-negative and anaerobic bacteria remains indicated. Besides adapting the empirically initiated antimicrobial therapy, culture results provide insights into local epidemiological patterns, which can be valuable in selecting the proper agents in the empirical phase.

Blood cultures are generally not recommended as they usually do not provide additional valuable information, especially in community-acquired cases. However, in cases with severe sepsis or septic shock, its use is mandatory. ${ }^{[16]}$ Also, blood cultures positive for anaerobic bacteria, especially Bacteroides fragilis, in sepsis of unclear origin, are a strong indication of an intra-abdominal focus. ${ }^{[17]}$ Systemic breakthrough as evidenced by positive blood culture represents fulminant infection, with a particularly grim prognosis. ${ }^{[18]}$

\section{Location of Primary Source and Microbiology}

Location of the primary source of secondary peritonitis influences the spectrum of pathogens involved, as stomach, upper small bowel, lower small bowel and large bowel have a distinct flora in terms of microbial species and density. Figure 1 summarizes initial culture results, according to the primary source of the IAI as found by de Ruiter et al. ${ }^{[19]}$ There exists a preponderance of Gram-negative and anaerobic bacteria in IAI originating from colorectal sources or appendicitis. In gastro-duodenal perforation, Gram-positive bacteria and yeasts are isolated most frequently. 
In IAI from a small intestine source, there is a relative balance between the four different groups of pathogenic micro-organisms. However, in subsequent weeks there is a shift in culture results, showing an increase in Gram-positive bacteria, while the prevalence of Gram-negatives decreases. ${ }^{[19]}$

Healthcare-Associated vs Community-Acquired IAI

Healthcare-associated infection includes all circumstances in which patients have had close association with either acute or chronic care settings. ${ }^{[20]}$ The relevance of the issue is that healthcare-associated IAI is inherently associated with an increased risk of resistant pathogen involvement. Based on a 10-year single-centre study of over 2000 cases of complicated IAI, Swenson et al. ${ }^{[21]}$ found that resistant pathogens were isolated from $79 \%$ of healthcare-associated infections. The risk of involvement of resistant pathogens is, however, also the result of exposure to antibacterials, irrespective of having close association with healthcare facilities as mentioned in the definitions. ${ }^{[20]}$ However, it should be mentioned that, in IAI occurring within the first days of hospitalization, the prevalence of resistant pathogens is not dramatically increased. ${ }^{[21,22]}$ In
Swenson et al., ${ }^{[21]}$ the average time between hospital admission and treatment of resistant pathogens was 10 days (13 days for non-fermenting Gramnegatives, 8 days for resistant staphylococci, 23 days for vancomycin-resistant enterococci [VRE] and 7 days for fungi). Also important, the average time from admission to treatment of nonresistant pathogens was still 5 days. Assuming that in these cases resistant pathogens were also cultured after about 5-7 days, these data indicate a timeframe within which resistant pathogens do not necessarily need to be covered in the empirical regimen. Of course, local epidemiological patterns should always be taken into account (e.g. quinolone resistance in Escherichia coli in the community). In nosocomial pneumonia, the risk of resistant pathogen involvement is also substantial after a hospitalization of about 5-7 days, leading to the concept of early-onset and lateonset infection. ${ }^{[23]}$ In IAI, however, such a concept, although probably of value in the context of empirical choices within antimicrobials, is not proposed in the current guidelines, in which no difference is made in risk assessment of healthcare-associated IAI according to length of hospitalization. ${ }^{[2]}$ In the proposed grid (table I), a

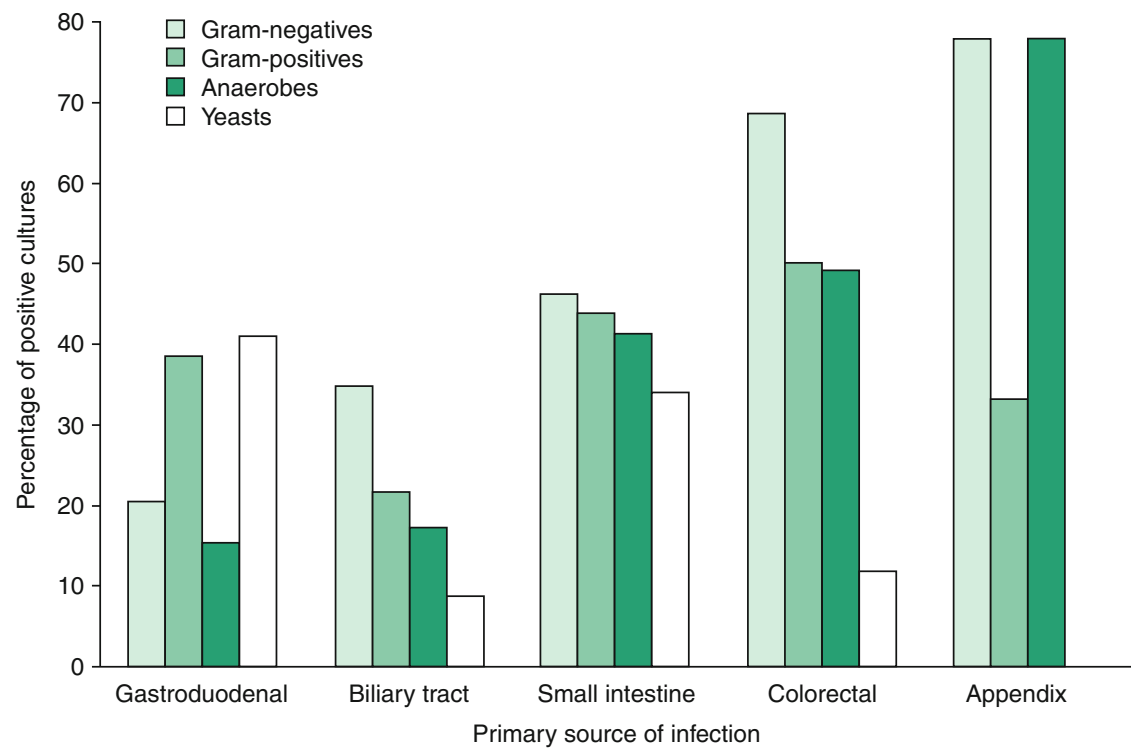

Fig. 1. Initial culture results in secondary and tertiary peritonitis: percentages of positive cultures according to the primary source of infection. ${ }^{[19]}$ 
distinction is made between community-acquired or early-onset healthcare-associated IAI $(<5-7$ days) and late-onset healthcare-associated IAI. Yet, recent exposure to antimicrobial agents must be considered as a risk factor for resistance, irrespective of origin of onset. Therefore, when it comes to selecting an empirical antimicrobial regimen, cases with community-onset IAI with recent antimicrobial exposure ( $\geq 2$ days of therapy) are categorized together with late-onset healthcare-associated IAI.

\section{Local Epidemiology of Resistance}

Empirical choices of antimicrobial agents are based upon the risk assessment of involvement of resistant pathogens. In IAI literature, the concept of resistance goes beyond in vitro multidrug resistance and basically includes all pathogens not essentially covered by first-line empirical regimens. As such, the following micro-organisms are considered 'resistant': methicillin-resistant Staphylococcus aureus (MRSA), coagulase-negative staphylococci, VRE, ESBL-producing Enterobacteriaceae, quinolone-resistant $E$. coli, nonfermenting Gram-negative bacteria (including P. aeruginosa, Stenotrophomonas maltophilia, Acinetobacter baumannii) and yeasts.

Although the ongoing emergence of resistance is a global concern, it is local epidemiology data that should be taken into account as they can substantially differ from the national average. ${ }^{[25]}$ For example, in a general hospital in Houston (TX, USA), the prevalence of fluoroquinoloneresistant E. coli was 2- to 4-fold higher than the national averages. ${ }^{[25]}$ The increase of quinoloneresistant E. coli in community-acquired infections was mostly a matter of urinary isolates in male outpatients or in sputum samples from inpatients. Therefore, the extent to which this problem weighs on the specific aetiology of IAIs is uncertain. Large multicentre surveillance studies which focus on resistance are scarce in IAI and hence the true prevalence of resistance is hard to estimate. Regardless, it is unjustified to take national averages of 'clinical specimens' as a reference for aetiology in IAIs. Data from the European Antimicrobial Resistance Surveillance System indicate that, in France, about $22 \%$ of all clinical isolates of $S$. aureus tested $(\mathrm{n}=4720)$ were resistant to methicillin. ${ }^{[26]}$ In contrast, in a prospective study performed in 25 French centres, Montravers et al. ${ }^{[27]}$ found not a single $S$. aureus isolate from nosocomial IAI to be methicillin resistant. So, although obviously present in other types of infection, MRSA is not a common pathogen in secondary peritonitis. An identical observation can be made for VRE. Although enterococci are important pathogens in IAI, as the overall prevalence of VRE is low, the role of VRE is limited to exceptional cases with late disease and to risk groups such as complicated liver transplantation. ${ }^{[22,27-29]}$ As such, in the majority of IAI, including community-acquired and early secondary peritonitis, enterococci and, in particular circumstances, VRE, do not need to be covered in the empirical regimen.

The need to cover ESBL Enterobacteriaceae in community-acquired IAI is also strongly dependent on geographic ecology. There are clear signals of epidemiological indications of expansion of ESBL E. coli and Klebsiella species in the community. This may be provoked by excessive use of antimicrobials in medical practice but also by the widespread contamination of the food chain with ESBL Enterobacteriaceae, which is also the case in countries with - until now - no extreme resistance problems, such as Denmark. ${ }^{[30-32]}$ Data from India indicate that more than half of all E. coli strains and about one-third of Klebsiella strains isolated from IAI produce ESBL. ${ }^{[33]}$ Data from Hawser et al. ${ }^{[33]}$ show important differences in resistance rates for E. coli and Klebsiella species among European countries, albeit that the study is unclear about the origin of infection (either community or hospital onset). Resistance rates for E. coli were highest in Turkey, Greece and Portugal, while fewer resistance problems were noted in France, Lithuania and Estonia. For Klebsiella, carbapemens appear to be a good choice for all European countries (more than 90\% susceptibility), with the exception of Greece, where only $43 \%$ of isolates were susceptible for both imipenem and ertapenem. In particular, because of the huge geographic variation in resistance rates, special attention should be given to patients with a recent history of travelling in regions 
known to have particular resistance problems, such as India, Thailand or Egypt. ${ }^{[34,35]}$ However, the extent to which antibacterial resistance in Enterobacteriaceae results in worse clinical outcomes in cases of community-acquired IAI, especially in circumstances where adequate surgical source control was promptly achieved, remains unknown.

Finally, $P$. aeruginosa is also a feared pathogen, the importance of which is frequently overestimated in the context of IAI. P. aeruginosa is a typically opportunistic pathogen, generally affecting immunocompromized or otherwise severely debilitated patients. Given the relatively poor ability of this micro-organism to invade tissue, the isolation of $P$. aeruginosa often represents contamination rather than true pathogen, especially in cases in which surgical intervention was promptly performed and infection, as such, was not established. In the large study by Swenson et al., ${ }^{[21]}$ non-fermenting Gram-negative bacteria (P. aeruginosa, S. maltophilia and A. baumannii) accounted for fewer than $6 \%$ of all IAIs. In a series of secondary peritonitis, $P$. aeruginosa accounted for about $9 \%$ of infections. Edelsberg et al. ${ }^{[36]}$ demonstrated that, in both community-acquired and healthcare-associated IAI, coverage of Pseudomonas did not add to higher rates of clinical success, indicating the limited importance of these pathogens. Yet, in patients with a particular risk profile for opportunistic pathogens (e.g. immunocompromized patients), coverage of Pseudomonas is warranted in all cases. ${ }^{[37]}$

\section{Coverage Against Enterococci}

Enterococcal involvement in IAI is generally encountered in patients with a loaded medical history and is associated with a grim prognosis. ${ }^{[38-40]}$ Routine coverage against enterococci is not mandatory in community-acquired IAI as it appears not to give additional benefit. ${ }^{[41-43]}$ Empirical coverage of enterococci is warranted in nosocomial IAI, which, in the majority, represents cases in whom early surgical source control was not achieved or complications such as anastomotic leakage occur. Reasonable indications for enterococcal coverage include septic shock, prolonged treatment with cephalosporins, immuno- suppression, presence of prosthetic heart valves and recurrent IAI with associated severe sepsis. ${ }^{[44-47]}$ When enterococcal infections are suspected, coverage should be included in the empirical regimen. Empirical therapy should primarily target Enterococcus faecalis. ${ }^{[24]}$ This may require additional drugs such as ampicillin or vancomycin when cephalosporin-based regimens are prescribed. In patients with a very high risk profile (e.g. a liver transplant patient with an IAI originating from the hepatobiliary tree or a patient known to be colonized with VRE) coverage of Enterococcus faecium is recommended. In this case, tigecycline or linezolid can be prescribed. ${ }^{[48,49]}$

\section{Coverage of Methicillin-Resistant Staphylococcus Aureus (MRSA)}

The presence of MRSA may either be based on pre-infection colonization or acquisition in the hospital. MRSA is often isolated in patients with long-standing, difficult-to-treat infections such as tertiary peritonitis. Vancomycin has been used most extensively in patients with intra-abdominal MRSA infection, but other drugs with demonstrated anti-MRSA activity, such as linezolid, tigecycline, daptomycin or quinupristin/dalfopristin, can be used Clinical experience in this category of severely ill patients is limited.

\section{Coverage Against Candida Species}

With the exception of patients with a particular risk profile for fungal infections (e.g. neutropenic patients) or patients with anastomotic leakage, Candida should not be covered by the empirical regimen. ${ }^{[50,51]}$ Even when initial culture results show Candida spp., the clinical relevance of these isolations remains vague and probably not all patients need antifungal coverage. ${ }^{[52]}$ In a prospective cohort of 62 patients with peritonitis following peptic ulcer perforations, Candida was isolated from $37 \%$ of cases $(n=23) \cdot{ }^{[53]}$ Antifungal therapy was initiated in eight patients, of whom only three survived. Yet, all patients in whom no antifungal therapy was initiated survived. This illustrates the ambiguous role of Candida as a pathogen in IAI.

An algorithm to determine which patients may benefit from antifungal therapy has been proposed. ${ }^{[54]}$ This algorithm is based on (i) presence 
or absence of established peritonitis; (ii) isolation of Candida spp.; (iii) presence or absence of severe sepsis or septic shock; and (iv) immunocompromized status. This algorithm, however, has not yet been subject to clinical evaluation. Invasive candidiasis is nearly always preceded by overt Candida colonization, and although the positive predictive value of prior colonization is rather low, failure to cover Candida species in the empirical phase is associated with treatment failure and death. ${ }^{[55,56]}$ Therefore, in cases of overt Candida colonization prior to infection onset, or when peri-operative cultures reveal Candida species, antifungal therapy is recommended. Dupont et al. ${ }^{[57]}$ developed and validated a simple score to estimate the likelihood of Candida involvement in peritonitis. This score describes risk levels of Candida involvement based on the presence or absence of four risk factors: female gender, upper gastrointestinal tract origin of peritonitis, peri-operative cardiovascular failure and previous antimicrobial therapy. The risk of Candida involvement is minimal when no or only one risk factor is present and maximal when all four are present. In the presence of three risk factors, the score had $84 \%$ sensitivity, but the $50 \%$ specificity was rather low, thereby limiting its usefulness in clinical practice.

Previous antibacterial therapy may also result in selection of fungi. With regard to Candida spp., prior exposure to fluconazole may select for nonalbicans Candida spp. with potentially reduced susceptibility to this agent. ${ }^{[58]}$ In these cases, an echinocandin is recommended. ${ }^{[59]}$

\subsection{Patient-Related Factors}

Recent guidelines for the diagnosis and treatment of complicated IAI make use of the concept 'high-risk patient'. ${ }^{[24]}$ This concept encompasses different risk factors for treatment failure and poor outcome. The prognosis of IAI depends on the overall quality of the management of infection, which includes aspects such as timely recognition of signs and symptoms, physiological resuscitation and organ support, adequate surgical source control and prompt initiation of appropriate antimicrobial therapy. Delays in initial interven-
Table IV. Factors contributing to a high-risk profile in intra-abdominal infections

\begin{tabular}{|c|c|}
\hline Factors & Associated risk \\
\hline \multicolumn{2}{|l|}{ Underlying conditions } \\
\hline $\begin{array}{l}\text { Older age ( }>70 \text { years) } \\
\text { Immunocompromized state } \\
\text { (pulmonary disease, liver } \\
\text { disease, transplantation, } \\
\text { etc.) }\end{array}$ & $\begin{array}{l}\text { Diminished inflammatory response } \\
\text { implying a risk of delayed recognition of } \\
\text { signs and symptoms with delayed } \\
\text { surgical intervention and initiation of } \\
\text { antimicrobial therapy }\end{array}$ \\
\hline $\begin{array}{l}\text { Prior antimicrobial } \\
\text { exposure }\end{array}$ & $\begin{array}{l}\text { Higher risk of involvement of multidrug- } \\
\text { resistant pathogens and/or yeasts }\end{array}$ \\
\hline
\end{tabular}

\section{Acute conditions}

High degree of intraabdominal contamination

Severe sepsis/septic shock

Difficult source control; inability to completely clean the peritoneal cavity Increased distribution volume and renal clearance, implying a risk of insufficient dosing of antimicrobial agents with inadequate microbial killing

tion and inability to achieve adequate debridement or drainage because of the high degree of peritoneal contamination are associated with failure of source control for IAI. ${ }^{[3,4]}$ Besides aspects of infection management, patient-related factors can also compromise the odds of survival. Table IV summarizes conditions leading to an increased risk of inadequate management and subsequent death. A distinction has to be made between underlying conditions and acute conditions, or severity of disease expression.

\subsection{Underlying Co-Morbidities}

Older age, low albumin level and poor nutritional status are risk factors for death following failure of source control. ${ }^{[1,21,24,60-62]}$ Yet, relationships between particular underlying conditions, involvement of resistant pathogens, inappropriate antimicrobial therapy and subsequent death have not been demonstrated so far. According to a large single-centre study including 2049 patients, complicated IAIs caused by multidrug-resistant pathogens are associated with higher mortality rates $(17.0 \%$ vs $8.6 \%$; odds ratio 2.19 , $95 \% \mathrm{CI}$ $1.53,3.13) .{ }^{[21]}$ Underlying conditions associated with the involvement of resistant pathogens were current corticosteroid use, solid organ transplant, and pulmonary and liver disease. However, these findings were not adjusted for previous antimicrobial exposure, the major risk factor for 
resistance. To the best of our knowledge, no underlying condition (as such HIV, chronic obstructive pulmonary disease, etc.) constitutes an increased risk of involvement of resistant pathogens. Therefore, the decision to cover resistant pathogens should essentially be based on the presence of major risk factors as indicated in the grid: recent antimicrobial exposure and/or lateonset healthcare-associated IAI.

\subsubsection{Penicillin Allergy}

Patients with proven allergy to penicillin should not receive ampicillin or its derivates such as amoxicillin or piperacillin/tazobactam. In cases of non-IgE-mediated allergy, cross-allergy is uncommon with cephalosporins and carbapenems, and never observed with fluoroquinolones, aminoglycosides, glycopeptides, imidazoles and linezolid. If the proposed regimen includes a penicillin derivate, it is better replaced by a cephalosporin or carbapenem. One should take into account, however, that cephalosporins have no activity against enterococci and anaerobic Gram-negative bacteria and that ertapenem does not cover Pseudomonas species. When indicated, a glycopeptide for enterococci or an imidazole for anaerobic bacteria must be prescribed. In IgEmediated allergy, cross-reaction with cephalosporins is rare. Nevertheless, these agents should be avoided. The drug of choice in such cases is aztreonam (combined with a glycopeptide and, if necessary, an imidazole) or a fluoroquinolone (combined with an imidazole if considered necessary). ${ }^{[63,64]}$ Tigecycline is another valuable option in penicillin-allergic patients. ${ }^{[65]}$ Additional coverage of $P$. aeruginosa is, however, necessary in patients at risk for Pseudomonas involvement.

\subsubsection{Severity of Disease Expression}

Severity of disease expression refers to the systemic inflammatory reaction provoked by the IAI and associated organ derangements. The proposed grid differentiates between mild, moderate and severe disease expression. Basically, this correlates with the traditional sepsis classification, respectively, sepsis, severe sepsis and septic shock. ${ }^{[66]}$ Hereby, sepsis is a condition characterized by a systemic inflammatory response syndrome, severe sepsis is accompanied by additional organ failure, and septic shock refers to a condition associated with arterial hypotension. The habit is to prescribe more last-line agents (typically carbapenems) in more pronounced disease expression, albeit that there is little rationale to support this practice. There is no reason to assume resistant pathogens on the basis of disease expression. As such, in the absence of true risk factors for resistance (late-onset healthcare-associated IAI and recent antimicrobial exposure), last-line antimicrobial agents cannot be recommended. However, one can assume that, in patients with moderate to severe disease expression, the margin by which one can afford inappropriate therapy is much smaller than in patients presenting with mild disease. It seems logical that the clinical consequences of inappropriate therapy will be more serious in patients with septic shock.

Using these elements to consider in selecting empirical antimicrobial therapy, a list of regimens is proposed by us. Each of these regimens falls back on the classification of IAI based on severity of disease expression (mild, moderate, severe), anatomical disruption, and community-acquired or early-onset healthcare-associated origin versus late-onset healthcare-associated origin and/or recent antimicrobial exposure (2-4 days of therapy) [table II]. Essentially, three regimens are proposed: (i) a baseline regimen without coverage of nosocomial Gram-negatives (including P. aeruginosa), enterococci or Candida species; (ii) a regimen with additional coverage of nosocomial Gram-negatives and enterococci; and (iii) a regimen with coverage of nosocomial Gram-negatives, enterococci or Candida species.

The proposed regimens are partially derived from the guidelines for the diagnosis and management of complicated IAIs and the guidelines for the treatment of invasive candidiasis. ${ }^{[24,59]}$ One should realize, however, that baseline regimens often cover a broader spectrum of pathogens than strictly needed (e.g. ertapenem, tigecycline or moxifloxacin). Yet, in order not to endanger the chances of appropriate therapy, antibacterial restriction should rather be achieved through correct indications for antimicrobial therapy and avoidance of unnecessarily long duration of therapy. 


\section{Recently Introduced Agents}

Recently, a number of newer drugs in different classes have been evaluated in the treatment of complicated IAI. These further expand the therapeutic options and further simplify treatment by reducing the number of administrations per day.

\subsection{Doripenem}

Doripenem is the newest carbapenem with a spectrum similar to that of meropenem and was approved by the US FDA for the treatment of complicated IAI in 2007. In a large randomized controlled trial, it was found to be non-inferior to meropenem. ${ }^{[67]}$ Compared with imipenem/ cilastatin, ${ }^{[68]}$ doripenem has been reported to have increased activity to a number of Gramnegative bacteria and also emergence of resistance during treatment was found to be lower, but the clinical impact of these findings is unclear. Doripenem dose in the complicated IAI studies was $500 \mathrm{mg}$ three times daily. Following its activity against Pseudomonas and ESBL-producing Enterobacteriaceae, doripenem is useful when these pathogens are a concern (table II).

\subsection{Moxifloxacin}

Moxifloxacin is a broad-spectrum fluoroquinolone that has recently been positioned as first-line agent in the treatment of IAI. It penetrates well and accumulates in gastrointestinal tissues and peritoneal fluid, ${ }^{[69]}$ and has demonstrated in vitro activity against the majority of organisms involved in complicated IAIs. ${ }^{[70]}$ It has been compared with several comparators, including $\beta$-lactam/lactamase inhibitors, cephalosporin-based therapy and carbapenems. ${ }^{[71-73]}$ In all these studies, non-inferiority with the comparator was found. Although an increase in resistance to moxifloxacin has been described in selected patients, this seems to be variable enough not to affect clinical efficacy. Yet it is prudent to evaluate the local resistance patterns when selecting empirical therapy for complicated IAI. Moxifloxacin is dosed at $400 \mathrm{mg}$ once daily. Moxifloxacin is best positioned as first-line singleagent therapy in circumstances where fluoro- quinolone-resistance of $E$. coli is not an issue (table II).

\subsection{Tigecycline}

Tigecycline belongs to a newer class of antibacterials, the glycylcyclines, and had in vitro activity against the flora commonly isolated in community-acquired complicated IAI. ${ }^{[7]}$ Its spectrum does not include Pseudomonas and therefore this drug is preferably positioned as first-line single-agent therapy (table II). On the other hand, tigecycline is active against MRSA, which can be advantageous in areas with a high prevalence of MRSA. The efficacy of tigecycline was comparable to that of meropenem in a large cohort of patients with complicated IAI. ${ }^{[75]} \mathrm{A}$ drawback, however, is that a pooled analysis of 13 clinical trials for both approved and unapproved indications for tigecycline (including one trial on complicated IAI), demonstrated an increased risk of death among patients receiving tigecycline compared with patients receiving comparator antibacterials (adjusted risk difference for all-cause mortality $0.6 \%$, 95\% CI 0.1 , 1.2). This observation led to a recommendation against the use of tigecycline in severe infections. ${ }^{[76]}$ Following this FDA drug safety communication, we do not recommend tigecycline in patients with IAI presenting with septic shock (tables I and II).

Tigecycline dosing is $50 \mathrm{mg}$ twice daily after a $100 \mathrm{mg}$ loading dose. It is a valuable option for patients with penicillin allergy.

\section{Directed Therapy}

Once the result of cultures obtained during surgery or after percutaneous drainage are available, de-escalation of antimicrobial therapy can be considered when broad-spectrum agents have been used as empirical therapy, most notably in patients with severe community-acquired disease. It should be remembered that anaerobes are more difficult to culture and that often only the most abundant micro-organisms are documented. As most complicated IAI are polymicrobial in nature, antimicrobial regimens used in a de-escalation 
Table V. Duration of antimicrobial therapy according to primary site of infection ${ }^{[80]}$

\begin{tabular}{|c|c|c|}
\hline Infection site & Clinical context & Duration of therapy \\
\hline Abscess & Exclusive liver or spleen abscesses & 3-7 days after surgery or drainage \\
\hline Acalculous cholecystitis & Percutanous drainage & 1-7 days according to clinical response \\
\hline Appendicitis & $\begin{array}{l}\text { No perforation } \\
\text { Gangrenous } \\
\text { Perforation }\end{array}$ & $\begin{array}{l}\text { Only peri-operative prophylaxis } \\
1-3 \text { days } \\
3-7 \text { days }\end{array}$ \\
\hline Ascending cholangitis & $\begin{array}{l}\text { No device } \\
\text { Device }\end{array}$ & $\begin{array}{l}\text { Up to } 24 \text { hours after drainage } \\
7 \text { days in moderate to severe cases } \\
\text { Prolonged therapy if case of liver abscess } \\
5 \text { days after drainage and device removal }\end{array}$ \\
\hline \multirow[t]{2}{*}{ Cholecystitis } & $\begin{array}{l}\text { Non-operative approach } \\
\text { Surgical approach }\end{array}$ & $5-10$ days \\
\hline & $\begin{array}{l}\text { - no perforation } \\
\text { - perforation }\end{array}$ & $\begin{array}{l}\text { - up to } 24 \text { hours } \\
\text { - } 5 \text { days }\end{array}$ \\
\hline Diverticulitis & $\begin{array}{l}\text { No perforation } \\
\text { Perforation }\end{array}$ & $\begin{array}{l}5-7 \text { days } \\
3-7 \text { days }\end{array}$ \\
\hline Gastro-duodenal perforation & $\begin{array}{l}\text { Time to intervention } \\
-<24 \text { hours } \\
->24 \text { hours }\end{array}$ & $\begin{array}{l}\text { - Peri-operative prophylaxis only } \\
\text { - 3-7 days }\end{array}$ \\
\hline Pancreatitis & $\begin{array}{l}\text { Proven infection or after } 10 \text { days of multiple } \\
\text { organ dysfunction }\end{array}$ & 3-7 days \\
\hline Peritonitis/anastomotic leakage & Localized or generalized & 3-7 days \\
\hline
\end{tabular}

strategy should also cover the full spectrum of aerobic and anaerobic micro-organisms, not only the micro-organisms that have been identified. The only exception to this rule may be patients with primary peritonitis, or selected patients with postoperative abscesses with only one pathogen identified on direct examination and culture.

\section{Duration of Antimicrobial Therapy for IAI}

The duration of antimicrobial therapy should ideally be defined from the start of the treatment. In case of complicated IAI with prompt surgical intervention and adequate source control, antimicrobial therapy is generally recommended to continue for 5-7 days, but shorter courses have been proposed, most often based on the extent of the infection and clinical response of the patient to the treatment. ${ }^{[77]}$ Yet, strong evidence to support such shorter courses remains scarce, especially for more severe cases. The largest body of evidence is on the duration of antimicrobials in children with acute appendicitis, where 3 days of antimicrobials seems sufficient. ${ }^{[78]} \mathrm{A}$ recent study found that adult patients with communityacquired localized peritonitis of mild to moderate severity who were treated for 3 days had similar outcomes to patients treated for 5-10 days. ${ }^{[79]}$ Besides this study, the proposed duration of antimicrobial therapy in IAI is largely based on expert opinion. Table V summarizes the proposed duration of antimicrobial therapy in IAI according to the primary source of infection. ${ }^{[80]}$ In patients who require (repetitive) reoperation because of failed source control, the operation in which the source is definitely controlled should be regarded as the index operation from which the duration of therapy is calculated. In selected patients, prolonged antimicrobial therapy may be necessary, especially when source control is difficult or impossible to obtain.

\section{Conclusion}

We proposed an alternative classification of IAI based on (i) anatomical disruption; (ii) severity of disease expression; and (iii) either communityacquired/early-onset healthcare-associated origin, or late-onset healthcare-associated origin and/or 
recent antimicrobial exposure. All possible combinations according to these three components are summarized in a grid. The grid allows physicians to select the most convenient empirical antimicrobial regimen according to the clinical situation. More precisely, it advises on the necessity of covering $P$. aeruginosa or resistant Gramnegative bacteria, enterococci and yeasts, albeit that local patterns of microbial ecology should also always be taken into account. In recent years, some newer agents such as doripenem, moxifloxacin and tigecycline have been added to the antibacterial armamentarium for IAI. While moxifloxacin and tigecycline can be used as firstline single-agent therapy, doripenem can be used when Pseudomonas and or resistant Gramnegative pathogens are a concern. In patients in whom the source can be adequately controlled, antimicrobial therapy should be restricted to a short course, generally 3-7 days after surgery.

\section{Acknowledgements}

This paper was prepared without funding. Jan J. De Waele has acted as a consultant for AstraZeneca, Bayer Healthcare and Wyeth. Neither of the other authors have any conflict of interest to disclose.

\section{References}

1. Blot S, De Waele JJ. Critical issues in the clinical management of complicated intra-abdominal infections. Drugs 2005; 65 (12): 1611-20

2. Schein M, Marshall J. Source control for surgical infections. World J Surg 2004; 28: 638-45

3. Singh R, Kumar N, Bhattacharya A, et al. Preoperative predictors of mortality in adult patients with perforation peritonitis. Indian J Crit Care Med 2011; 15 (3): $157-63$

4. De Waele JJ. Early source control in sepsis. Langenbecks Arch Surg 2010; 395 (5): 489-94

5. Schein M, Marshall J. Source control: a guide to the management of surgical infections. Heidelberg: Springer Verlag, 2002

6. Evans HL, Raymond DP, Pelletier SJ, et al. Diagnosis of intra-abdominal infection in the critically ill patient. Curr Opin Crit Care 2001; 7: 117-21

7. Vincent JL, Rello J, Marshall J, et al. International study of the prevalence and outcomes of infection in intensive care units. JAMA 2009; 302: 2323-9

8. Chromik AM, Meiser A, Holling J, et al. Identification of patients at risk for development of tertiary peritonitis on a surgical intensive care unit. J Gastrointest Surg 2009; 13 (7): $1358-67$
9. Solomkin JS, Hemsell DL, Sweet R, et al. Evaluation of new anti-infective drugs for the treatment of intraabdominal infections. Infectious Diseases Society of America and the Food and Drug Administration. Clin Infect Dis 1992; 15 Suppl. 1: S33-42

10. Knaus WA, Draper EA, Wagner DP, et al. APACHE II: a severity of disease classification system. Crit Care Med 1985; 13: 818-29

11. Berger D, Buttenschoen K. Management of abdominal sepsis. Langenbecks Arch Surg 1998; 383: 35-43

12. Gauzit R, Pean Y, Barth X, et al. Epidemiology, management, and prognosis of secondary non-postoperative peritonitis: a French prospective observational multicenter study. Surg Infect (Larchmt) 2009; 10: 119-27

13. Leclercq R. Epidemiological and resistance issues in multidrug-resistant staphylococci and enterococci. Clin Microbiol Infect 2009; 15: 224-31

14. Hecht DW. Prevalence of antibiotic resistance in anaerobic bacteria: worrisome developments. Clin Infect Dis 2004; 39: $92-7$

15. Dougherty SH. Antimicrobial culture and susceptibility testing has little value for routine management of secondary bacterial peritonitis. Clin Infect Dis 1997; 25 Suppl. 2: S258-61

16. Dellinger RP, Carlet JM, Masur H, et al. Surviving sepsis campaign guidelines for management of severe sepsis and septic shock. Intensive Care Med 2004; 30: 536-55

17. Lawrence PF, Tietjen GW, Gingrich S, et al. Bacteroides bacteremia. Ann Surg 1977; 186: 559-63

18. De Waele JJ, Hoste EA, Blot SI. Blood stream infections of abdominal origin in the intensive care unit: characteristics and determinants of death. Surg Infect (Larchmt) 2008; 9: 171-7

19. de Ruiter J, Weel J, Manusama E, et al. The epidemiology of intra-abdominal flora in critically ill patients with secondary and tertiary abdominal sepsis. Infection 2009; 37: 522-7

20. Klevens RM, Morrison MA, Nadle J, et al. Invasive methicillin-resistant Staphylococcus aureus infections in the United States. JAMA 2007; 298: 1763-71

21. Swenson BR, Metzger R, Hedrick TL, et al. Choosing antibiotics for intra-abdominal infections: what do we mean by "high risk"? Surg Infect (Larchmt) 2009; 10: 29-39

22. Seguin P, Laviolle B, Chanavaz C, et al. Factors associated with multidrug-resistant bacteria in secondary peritonitis: impact on antibiotic therapy. Clin Microbiol Infect 2006; 12: $980-5$

23. Vogelaers D, De Bels D, Foret F, et al. Patterns of antimicrobial therapy in severe nosocomial infections: empiric choices, proportion of appropriate therapy, and adaptation rates. A multicentre, observational survey in critically ill patients. Int J Antimicrob Agents 2010; 35: 375-81

24. Solomkin JS, Mazuski JE, Bradley JS, et al. Diagnosis and management of complicated intra-abdominal infection in adults and children: guidelines by the Surgical Infection Society and the Infectious Diseases Society of America. Clin Infect Dis 2010; 50: 133-64

25. Boyd LB, Atmar RL, Randall GL, et al. Increased fluoroquinolone resistance with time in Escherichia coli from $>17000$ patients at a large county hospital as a function of culture site, age, sex, and location. BMC Infect Dis 2008; 8: 4

26. European Centres for Disease Prevention and Control. Surveillance report: antimicrobial resistance surveillance in 
Europe 2009. Stockholm: European Centres for Disease Prevention and Control, 2009

27. Montravers P, Lepape A, Dubreuil L, et al. Clinical and microbiological profiles of community-acquired and nosocomial intra-abdominal infections: results of the French prospective, observational EBIIA study. J Antimicrob Chemother 2009; 63: 785-94

28. Augustin P, Kermarrec N, Muller-Serieys C, et al. Risk factors for multidrug resistant bacteria and optimization of empirical antibiotic therapy in postoperative peritonitis. Crit Care 2010; 14: R20

29. Cercenado E, Torroba L, Canton R, et al. Multicenter study evaluating the role of enterococci in secondary bacterial peritonitis. J Clin Microbiol 2010; 48: 456-9

30. Egea P, Lopez-Cerero L, Navarro MD, et al. Assessment of the presence of extended-spectrum beta-lactamase-producing Escherichia coli in eggshells and ready-to-eat products. Eur J Clin Microbiol Infect Dis 2011; 30 (9): 1045-7

31. Dolejska M, Matulova M, Kohoutova L, et al. Extendedspectrum beta-lactamase-producing Escherichia coli in turkey meat production farms in the Czech Republic: national survey reveals widespread isolates with bla(SHV-12) genes on IncFII plasmids. Lett Appl Microbiol 2011; 53: 271-7

32. Jensen VF. The development of antibiotics use in Danish food production [in Danish]. Ugeskr Laeg 2011; 173 (45): $2862-6$

33. Hawser SP, Badal RE, Bouchillon SK, et al. Antibiotic susceptibility of intra-abdominal infection isolates from Indian hospitals during 2008. J Med Microbiol 2010; 59: $1050-4$

34. Tham J, Odenholt I, Walder M, et al. Extended-spectrum beta-lactamase-producing Escherichia coli in patients with travellers' diarrhoea. Scand J Infect Dis 2010; 42: 275-80

35. Dhanji H, Patel R, Wall R, et al. Variation in the genetic environments of bla(CTX-M-15) in Escherichia coli from the faeces of travellers returning to the United Kingdom. J Antimicrob Chemother 2011; 66: 1005-12

36. Edelsberg J, Berger A, Schell S, et al. Economic consequences of failure of initial antibiotic therapy in hospitalized adults with complicated intra-abdominal infections. Surg Infect (Larchmt) 2008; 9: 335-47

37. Freifeld AG, Bow EJ, Sepkowitz KA, et al. Clinical practice guideline for the use of antimicrobial agents in neutropenic patients with cancer: 2010 update by the Infectious Diseases Society of America. Clin Infect Dis 2011; 52: e56-93

38. Dupont H, Friggeri A, Touzeau J, et al. Enterococci increase the morbidity and mortality associated with severe intraabdominal infections in elderly patients hospitalized in the intensive care unit. J Antimicrob Chemother 2011; 66: 2379-85

39. Riche FC, Dray X, Laisne MJ, et al. Factors associated with septic shock and mortality in generalized peritonitis: comparison between community-acquired and postoperative peritonitis. Crit Care 2009; 13: R99

40. Seguin P, Brianchon C, Launey Y, et al. Are enterococci playing a role in postoperative peritonitis in critically ill patients? Eur J Clin Microbiol Infect Dis. Epub 2011 Nov 12

41. Cohn SM, Lipsett PA, Buchman TG, et al. Comparison of intravenous/oral ciprofloxacin plus metronidazole versus piperacillin/tazobactam in the treatment of complicated intraabdominal infections. Ann Surg 2000; 232: 254-62

42. Ohlin B, Cederberg A, Forssell H, et al. Piperacillin/ tazobactam compared with cefuroxime/ metronidazole in the treatment of intra-abdominal infections. Eur J Surg 1999; 165: 875-84

43. Walker AP, Nichols RL, Wilson RF, et al. Efficacy of a beta-lactamase inhibitor combination for serious in traabdominal infections. Ann Surg 1993; 217: 115-21

44. Fernandez-Guerrero ML, Herrero L, Bellver M, et al. Nosocomial enterococcal endocarditis: a serious hazard for hospitalized patients with enterococcal bacteraemia. J Intern Med 2002; 252: 510-5

45. Harbarth S, Cosgrove S, Carmeli Y. Effects of antibiotics on nosocomial epidemiology of vancomycin-resistant enterococci. Antimicrob Agents Chemother 2002; 46: 1619-28

46. Harbarth S, Uckay I. Are there patients with peritonitis who require empiric therapy for enterococcus? Eur J Clin Microbiol Infect Dis 2004; 23: 73-7

47. Patel R, Badley AD, Larson-Keller J, et al. Relevance and risk factors of enterococcal bacteremia following liver transplantation. Transplantation 1996; 61: 1192-7

48. Swoboda S, Ober M, Hainer C, et al. Tigecycline for the treatment of patients with severe sepsis or septic shock: a drug use evaluation in a surgical intensive care unit. J Antimicrob Chemother 2008; 61: 729-33

49. Linden PK. Treatment options for vancomycin-resistant enterococcal infections. Drugs 2002; 62: 425-41

50. Blot S, Vandewoude K. Management of invasive candidiasis in critically ill patients. Drugs 2004; 64 (19): 2159-75

51. Eggimann P, Francioli P, Bille J, et al. Fluconazole prophylaxis prevents intra-abdominal candidiasis in high-risk surgical patients. Crit Care Med 1999; 27: 1066-72

52. Rex JH. Candida in the peritoneum: passenger or pathogen? Crit Care Med 2006; 34: 902-3

53. Lee SC, Fung CP, Chen HY, et al. Candida peritonitis due to peptic ulcer perforation: incidence rate, risk factors, prognosis and susceptibility to fluconazole and amphotericin B. Diagn Microbiol Infect Dis 2002; 44: 23-7

54. Blot S, Vandewoude K, De Waele J. Candida peritonitis. Curr Opin Crit Care 2007; 13: 195-9

55. Magill SS, Swoboda SM, Johnson EA, et al. The association between anatomic site of Candida colonization, invasive candidiasis, and mortality in critically ill surgical patients. Diagn Microbiol Infect Dis 2006; 55: 293-301

56. Agvald-Ohman C, Klingspor L, Hjelmqvist $\mathrm{H}$, et al. Invasive candidiasis in long-term patients at a multidisciplinary intensive care unit: candida colonization index, risk factors, treatment and outcome. Scand J Infect Dis 2008; 40: $145-53$

57. Dupont H, Bourichon A, Paugam-Burtz C, et al. Can yeast isolation in peritoneal fluid be predicted in intensive care unit patients with peritonitis? Critical Care Med 2003; 31: $752-7$

58. Blot S, Janssens R, Claeys G, et al. Effect of fluconazole consumption on long-term trends in candidal ecology. J Antimicrob Chemother 2006; 58: 474-7

59. Pappas PG, Kauffman CA, Andes D, et al. Clinical practice guidelines for the management of candidiasis: 2009 update 
by the Infectious Diseases Society of America. Clin Infect Dis 2009; 48: 503-35

60. Pine RW, Wertz MJ, Lennard ES, et al. Determinants of organ malfunction or death in patients with intraabdominal sepsis: a discriminant analysis. Arch Surg 1983; 118: $242-9$

61. Mulier S, Penninckx F, Verwaest C, et al. Factors affecting mortality in generalized postoperative peritonitis: multivariate analysis in 96 patients. World J Surg 2003; 27: $379-84$

62. Schneider CP, Seyboth C, Vilsmaier M, et al. Prognostic factors in critically ill patients suffering from secondary peritonitis: a retrospective, observational, survival time analysis. World J Surg 2009; 33: 34-43

63. Saxon A, Hassner A, Swabb EA, et al. Lack of crossreactivity between aztreonam, a monobactam antibiotic, and penicillin in penicillin-allergic subjects. J Infect Dis 1984; 149: 16-22

64. Saxon A, Beall GN, Rohr AS, et al. Immediate hypersensitivity reactions to beta-lactam antibiotics. Ann Intern Med 1987; 107: 204-15

65. Barberán J, Mensa J, Fariñas C, et al. Recommendations of antimicrobial treatment in patients allergic to beta-lactam antibiotics [in Spanish]. Rev Esp Quimioter 2008; 21 (1): 60-82

66. Bone RC. Let's agree on terminology: definitions of sepsis. Crit Care Med 1991; 19: 973-6

67. Lucasti C, Jasovich A, Umeh O, et al. Efficacy and tolerability of IV doripenem versus meropenem in adults with complicated intra-abdominal infection: a phase III, prospective, multicenter, randomized, double-blind, noninferiority study. Clin Ther 2008; 30: 868-83

68. Pillar CM, Torres MK, Brown NP, et al. In vitro activity of doripenem, a carbapenem for the treatment of challenging infections caused by Gram-negative bacteria, against recent clinical isolates from the United States. Antimicrob Agents Chemother 2008; 52: 4388-99

69. Wirtz M, Kleeff J, Swoboda S, et al. Moxifloxacin penetration into human gastrointestinal tissues. J Antimicrobial Chemother 2004; 53: 875-7

70. Edminston CE, Krepel CJ, Seabrook GR, et al. In vitro activities of moxifloxacin against 900 aerobic and anaerobic surgical isolates from patients with intra-abdominal and diabetic foot infections. Antimicrob Agents Chemother 2004; 48: 1012-6

71. Solomkin J, Zhao Y-P, Ma E-L, et al., DRAGON Study Team. Moxifloxacin is non-inferior to combination ther- apy with ceftriaxone plus metronidazole in patients with community-origin complicated intra-abdominal infections. Int J Antimicrob Agents 2009; 34: 439-45

72. Malangoni MA, Song J, Herrington J, et al. Randomized controlled trial of moxifloxacin compared with piperacillin-tazobactam and amoxicillin-clavulanate for the treatment of complicated intra-abdominal infections. Ann Surg 2006; 244: 204-11

73. De Waele J, Tellado J, Reimnitz P, et al. Efficacy and safety of moxifloxacin vs. ertapenem in complicated intraabdominal infections: results of the PROMISE study. Abstracts of the 20th European Congress of Clinical Microbiology and Infectious Diseases, Vienna, Austria, 10-13 April 2010. P1549

74. Gales AC, Jones RN. Antimicrobial activity and spectrum of the new glycylcycline, GAR-936 tested against 1,203 recent clinical bacterial isolates. Diagn Microbiol Infect Dis 2000; 36: 19-36

75. Babinchak T, Ellis-Grosse E, Dartois N, et al. The efficacy and safety of tigecycline for the treatment of complicated intra-abdominal infections: analysis of pooled clinical trial data. Clin Infect Dis 2005; 41 Suppl. 5: S354-67

76. US Food and Drug Administration. FDA drug safety communication: increased risk of death with Tygacil (tigecycline) compared to other antibiotics used to treat similar infections [online]. Available from URL: http:/ www.fda.gov/Drugs/DrugSafety/ucm224370.htm [Accessed 2012 Mar 8]

77. Schein M, Assalia A, Bachus H. Minimal antibiotic therapy after emergency abdominal surgery: a prospective study. $\mathrm{Br}$ J Surg 1994; 81: 989-91

78. Snelling CM, Poenaru D, Drover JW. Minimum postoperative antibiotic duration in advanced appendicitis in children: a review. Pediatr Surg Int 2004; 20: 838-45

79. Basoli A, Chirletti P, Cirino E, et al. A prospective, doubleblind, multicenter, randomized trial comparing ertapenem 3 vs $>$ or $=5$ days in community-acquired intraabdominal infection. J Gastrointest Surg 2008; 12 (3): 592-600

80. Laterre PF, Colardyn F, Delmee M, et al. Antimicrobial therapy for intra-abdominal infections: guidelines from the Infectious Diseases Advisory Board. Ghent: Mapu, 2006

Correspondence: Professor Dr S. Blot, General Internal Medicine \& Infectious Diseases, Ghent University Hospital, De Pintelaan 185, 9000 Ghent, Belgium.

E-mail: stijn.blot@UGent.be 